\title{
Avaliação da Polaridade Superficial de Náilons por Espectroscopia de Fluorescência
}

\author{
Leonardo D. C. Baldi, Teresa D. Z. Atvars \\ Instituto de Química, UNICAMP
}

\begin{abstract}
Resumo: Este trabalho avalia a polaridade superficial de cinco tipos de náilons: $-6,-11,-6,6,-6,10,-6,12$ usando a escala do pireno, py. Esses náilons podem ser classificados em duas categorias: AB (nálion-6 e -11) e AABB (nálion-6,6 e $-6,10$ e -6,12). A escala py está baseada nas propriedades fotofísicas do pireno, que é obtida usando-se espectroscopia fotoestacionária de fluorescência e espectroscopia de decaimento de fluorescência. A partir dos espectros de fluorescência foram determinadas as razões de intensidades das bandas vibrônicas, $\mathrm{I}_{\mathrm{I}} / \mathrm{I}_{\mathrm{III}}$, que aumentam com a polaridade do náilon (náilon-6 maior que náilon-11 e assim por diante). Observou-se também que o tempo de meia vida diminui com a polaridade, como esperado. A partir destes experimentos, mostrou-se que o pireno é um sensor de polaridade de curta distância, isto é de distância similar ao seu raio molecular. Discute-se ainda a vantagem desta metodologia que agrega a facilidade na preparação de amostras, porque medidas podem ser feitas em amostras na forma de pó, pérolas ou filmes, independentemente da espessura.
\end{abstract}

Palavras-chave: Pireno, espectroscopia de fluorescência, náilons, polaridade.

\section{Evaluation of Surface Polarity of Nylons by Fluorescence Spectroscopy}

Abstract: This work evaluates the surface polarity of five types of nylons: $-6,-6,6,-11,-6,10,-6,12$ using the $p y$-scale. These nylons can be classified in two categories: AB (nylon-6 and -11) or AABB (nylon 6,6; 6,10 and 6,12). The pyscale is based on the photophysical properties of pyrene, which is obtained using steady-state fluorescence spectroscopy or the lifetime of the singlet excite state. From the steady-state fluorescence spectra we determine the ratio of the intensities of the vibronic bands, $\mathrm{I}_{\mathrm{I}} / \mathrm{I}_{\mathrm{III}}$, which increase with the polymer polarity (nylon- 6 higher than nylon-11 and so on). We also observed that the lifetime decreases with the polarity, as expected. From these experiments we also showed that pyrene is a polarity sensor for short distances, i. e. similar to the molecular radius. We also discuss that the major advantage of these methodologies is the facility of the sample preparation because measurements can be performed with samples as powder, pellets or films (independently of the thickness).

Keywords: Pyrene, fluorescence spectroscopy, nylons, polarity sites.

\section{Introdução}

Diversos métodos espectroscópicos são usualmente empregados para a caracterização química de polímeros. Destacam-se os métodos de ressonância magnética nuclear ${ }^{[1]} \mathrm{e}$ a espectroscopia vibracional na região do infravermelho ${ }^{[2]}$. Vários outros métodos, entretanto, têm se mostrado úteis como técnicas complementares para entendimento da micromorfologia. Destacam-se entre eles: aniquilação de positrônio ${ }^{[3,4]}$, espectroscopia vibracional Raman e Raman ressonante ${ }^{[5]}$, espectroscopia eletrônica de absorção na região do ultravioleta e visível ${ }^{[6]}$, espectroscopia de luminescência com e sem resolução temporal ${ }^{[6]}$, diversos tipos de métodos fotoquímicos ${ }^{[7]}$ e métodos ópticos não-lineares ${ }^{[8-10]}$.

Nos casos das espectroscopias de fotoluminescência e dos métodos ópticos não-lineares, a condição essencial é que o material polimérico contenha um grupo ou uma molécula capaz de produzir sinal óptico (emissão de luz, mudança de índice de refração ou de absortividade molar, entre outros). Para produzir sinal de fotoemissão, é necessário que o polímero seja intrinsecamente luminescente; neste caso, as medidas podem ser diretamente realizadas com o material. No caso deste não apresentar luminescência intrínseca, algumas estratégias podem ser priorizados: modificação química acrescentando um grupo ou segmento luminescente ${ }^{[11,12]}$; copolimerização com grupos ou monômeros luminescentes ${ }^{[13]}$; adsorção de uma molécula com a propriedade desejada ${ }^{[14-19]}$. Esse último método é muito conveniente por sua simplicidade, mas apresenta dificuldades: há dependência da solubilidade da molécula sensora na matriz polimérica podendo, portanto, ocorrer perdas por pervaporação ${ }^{[20]}$.

Vários métodos podem ser empregados na adsorção de moléculas luminescentes na matriz polimérica: adsorção

Autor para correspondência: Teresa D. Z. Atvars, Instituto de Química, UNICAMP, Caixa Postal 6154, CEP: 13084-970, Campinas, SP. E-mail: tatvars@iqm.unicamp.br 
por intumescimento utilizando um não-solvente do polímero ${ }^{[14-19]}$, formação de filmes por espalhamento de soluções contendo o emissor e o polímero ${ }^{[10,13,14,17,18]}$, deposição a partir do vapor da sonda luminescente ${ }^{[15]}$. Em todos os casos, um bom controle da concentração deve ser efetuado para evitar ou minimizar os processos de transferências de energia intermoleculares, que criam complicações ao entendimento dos processos fotofísicos decorrentes da absorção do fóton de excitação ${ }^{[6,12,15,21,22]}$.

Uma das áreas para as quais entendimento dos processos de adsorção superficial de corantes é de fundamental interesse é o tingimento ou branqueamento de fibras sintéticas, naturais ou modificadas ${ }^{[23-25]}$. Nessa área, dois enfoques principais são discutidos: um, tecnológico, que envolve a determinação da quantidade de sorvato, a estabilidade das cores e a otimização de processos de tingimento; outro, que envolve simulações das interações corante-fibra ${ }^{[26]}$. Ainda é necessário muito esforço acadêmico para o entendimento das forças intermoleculares que controlam a eficiência do processo de adsorção, do coramento e do branqueamento ${ }^{[27]}$. Esse esforço inclui o desenvolvimento de metodologias sensíveis que possam determinar a natureza das forças de interação com a superfície que controlam a eficiência do processo de adsorção e, por conseqüência, da polaridade superficial. Uma metodologia que permite tal estudo é a espectroscopia de fluorescência de corantes adsorvidos superficialmente ${ }^{[19]}$. Para isto, é necessário o uso de moléculas fluorescentes sensíveis à polaridade do meio. Um exemplo delas é o pireno.

O pireno é um hidrocarboneto aromático catacondensado com alto rendimento quântico de fluorescência quando excitado com radiação ultravioleta. Sua sensibilidade às propriedades do meio que o rodeia é manifestada em seu espectro de fluorescência, que consiste de um conjunto complexo de bandas vibrônicas. As bem resolvidas são numeradas de I a $\mathrm{V}$ em ordem decrescente de energia ${ }^{[6,22,28,29]}$. Os valores da relação entre as intensidades das bandas I e III, $\mathrm{I}_{\mathrm{I}} / \mathrm{I}_{\mathrm{III}}$, formam a escala de polaridade $p y$ do pireno ${ }^{[28,29]}$. Para uma variedade de solventes, esses valores estão tabelados. O valor dessa relação aumenta quando a polaridade do meio é maior. Além disso, também o tempo de vida do estado eletrônico excitado diminui com o aumento da polaridade do meio no qual o pireno estiver inserido ${ }^{[6,22]}$, quer seja um solvente ${ }^{[28,29]}$, uma fase micelar ${ }^{[30]}$, um sólido inorgânico ${ }^{[31]}$, um polímero ou um copolímero $^{[12]}$.

Mostramos recentemente que, com o pireno, pode-se determinar a polaridade de uma série de copolímeros de etileno$c o$-acetato de vinila ${ }^{[12]}, \mathrm{EVA}$, com vários teores de acetato de vinila. Os estudos mostraram que, aumentando o conteúdo de acetato de vinila nos copolímeros de EVA, tornando o meio mais polar, a relação entre as intensidades $\mathrm{I}_{\mathrm{I}} / \mathrm{I}_{\mathrm{III}}$ do espectro de fluorescência se altera e também diminui o tempo de decaimento. Sendo os EVA copolímeros aleatórios produzidos por polimerização radicalar, não há controle sobre o tamanho e a distribuição dos blocos de etileno e acetato de vinila na cadeia. Assim, a sensibilidade do pireno à polaridade, com o aumento do conteúdo de acetato de vinila, repre- senta uma média dos diversos ambientes experimentados pela sonda. Em função disto, apesar da sensibilidade da fluorescência do pireno se relacionar diretamente com a quantidade de acetato de vinila presente, pouco se pode discutir sobre a geometria e as distâncias entre os grupos acetato e pireno a partir das quais a sensibilidade cessa.

Este trabalho tem como função permitir que se discuta os limites de sensibilidade dessa sonda de polaridade em termos espaciais e as dimensões prováveis dos sítios superficiais de adsorção em um substrato cuja polaridade pode ser variada a partir dos comprimentos dos segmentos alifáticos de um dos monômeros. Para isso escolhemos como sistemas diversos tipos de náilons, polímeros lineares obtidos por reações de condensação, cujo tamanho dos segmentos mais polares pode ser variado de acordo com o tipo de monômero empregado na polimerização ${ }^{[31]}$.

Náilons são polímeros semicristalinos que possuem em sua estrutura grupos amida e grupos carboxílicos ${ }^{[32]}$. A determinação de sua polaridade superficial decorre das aplicações desses materiais como forma de fibras têxteis que, ao serem submetidas a processos de coramento/branqueamento, exigem que fortes interações corante/substrato (fibra) ocor$\operatorname{ram}^{[23-25]}$. A determinação das propriedades superficiais desses substratos, como sua polaridade, avalia a possibilidade de uma boa interação corante/substrato. Para tanto será utilizada, neste trabalho, a espectroscopia de fluorescência do pireno adsorvida na superfície dos náilons.

Duas categorias deles são aqui utilizadas: o tipo $\mathrm{AB}$ (náilons-6 e -11), nos quais varia o comprimento da cadeia alifática, tornando-os menos polares, e o tipo AABB (náilons-6,6, -6,10 e -6,12), nos quais se está mantendo constante o comprimento da cadeia apolar alifática do monômero amino, aumentando-se o comprimento dos grupos carboxilícos. As estruturas correspondentes serão mostradas oportunamente. A polaridade superficial das amostras foi avaliada a partir dos valores da razão $\mathrm{I}_{\mathrm{I}} / \mathrm{I}_{\mathrm{III}}$ dos espectros de fluorescência em condições fotoestacionárias e do tempo de meia vida do estado eletrônico excitado. Esses valores se correlacionam com o comprimento dos eixos longos das moléculas de polímero e, através disto, com o tamanho do segmento dos grupos carboxílicos da cadeia dos polímeros. O tamanho da cadeia do monômero foi estimado através de cálculos semiempríricos utilizando-se o Hamiltoniano AM1 para a otimização da geometria ${ }^{[33]}$.

\section{Experimental}

As amostras de náilon-6, -11, -6,6, -6,10 e -6,12, na forma de pérolas, e o pireno, foram fornecidos pela Aldrich Chemical Co. e utilizados sem purificação. As amostras de náilon foram dissolvidas em ácido fórmico e secas em placa de Petri. As pérolas foram transformadas em pó, prensadas a frio e tornadas pastilhas, sobre cuja superfície o vapor de pireno foi depositado, aquecendo-se seus cristais a $50{ }^{\circ} \mathrm{C}$. O tempo de deposição foi controlado para que a emissão das amostras correspondesse apenas a moléculas isoladas ${ }^{[12]}$. 
Obtiveram-se os espectros de fluorescência em condições fotoestacionárias em um espectrofluorímetro ISS modelo PC1 operando com uma lâmpada de xenônio de $300 \mathrm{~W}$. Selecionou-se comprimento de onda de excitação em $\lambda_{\text {exc }}=337 \mathrm{~nm}$. Os espectros de fluorescência foram registrados entre 350-480 $\mathrm{nm}$. Os decaimentos de fluorescência foram obtidos em um espectrofluorímetro com resolução temporal FL 900 da Edinburg Analytical Instruments com detetor do tipo single photon counting. As amostras foram excitadas utilizando-se uma lâmpada pulsada de hidrogênio com freqüência de repetição de $40 \mathrm{kHz}$. Cada amostra de náilon foi colocada em uma cubeta de quartzo selada sob vácuo. As medidas do decaimento foram realizadas excitando-se a amostra com $\lambda_{\text {exc }}=337 \mathrm{~nm}$ e coletando-se o sinal de emissão em $\lambda_{\mathrm{em}}=393 \mathrm{~nm}$.

O sinal de decaimento observado R(t) em função do tempo é dado pela integral:

$$
R(t)=\int_{0}^{t} F\left(t-t^{\prime}\right) L\left(t^{\prime}\right) d t
$$

em que $\mathrm{L}(\mathrm{t}$ ') é o tempo do pulso da lâmpada e $\mathrm{F}(\mathrm{t})$ é a função de resposta correspondente a um tempo de pulso infinitamente curto. L(t) foi determinado usando Ludox-DuPont, uma solução com alto nível de espalhamento de luz, na mesma faixa espectral e temporal do sinal da amostra. Este dado é analisado através de um método numérico de série de exponenciais, que assume o sinal $\mathrm{F}(\mathrm{t})$ como podendo ser analisado por funções multiexponenciais:

$$
F(t)=\sum_{i=1}^{n} A_{i} \exp \left(-\frac{t}{\tau_{i}}\right)
$$

sendo $A_{i}$ um fator pré-exponencial representando a contribuição do tempo de decaimento $\tau_{\mathrm{i}}$ ao sinal total de decaimento da amostra. Usando-se a função (2) pode-se simular os dados experimentais o que é feito utilizando-se um programa desenvolvido pelo fabricante do instrumento, que utiliza um algoritmo de Marquardt para minimizar o valor de $\chi^{2}$. Esse valor próximo da unidade e uma distribuição aleatória de resíduos são os critérios utilizados para considerar um bom ajuste das curvas experimentais ${ }^{[12,13]}$.

Imagens de amostras contendo pireno adsorvido na superfície podem ser obtidas por microscopia óptica de epifluorescência. Para isso, utilizou-se um microscópio óptico invertido de fluorescência Leica DM IRB, operando com uma lâmpada de mercúrio de alta pressão (HBO-100 W) como fonte de excitação, selecionando-se os comprimentos de onda para excitação na região espectral do ultravioleta utilizando-se filtros ópticos para 330-380 nm. A imagem da amostra emitindo é separada da radiação de excitação através de um filtro de barreira (um espelho dicróico para $\lambda_{\mathrm{em}}>400 \mathrm{~nm}$ ). As imagens foram capturadas com uma câmara digital Samsung SDC311 interfaciada, processadas pelo programa Liksys for Windows Versão 2.38, com o microscópio operando na configuração de epifluorescência ${ }^{[13,34]}$. São comparadas imagens de amostras com e sem pireno adsorvido.

\section{Resultados e Discussão}

As amostras de náilon contendo pireno adsorvido em superfície foram analisadas por espectroscopia de fluorescência em condições fotoestacionárias e por microscopia óptica de fluorescência, a qual permite observar o azul característico da emissão de fluorescência do pireno. A amostra sem pireno não é fluorescente. As micrografias ópticas estão mostradas na Figura 1, para uma amostra de náilon com e outra sem pireno.

A cor azul observada corresponde à região espectral do visível entre 390-480 nm, conforme pode ser observado através dos espectros de fluorescência do pireno nos diferentes náilons (Figura 2). O espectro de fluorescência é composto por cinco bandas bem resolvidas, sobrepostas a um envelope largo, sendo cada uma denominada banda vibrônica ${ }^{[6,22,28,29]}$. As relações entre as intensidades das bandas I $\left(\lambda_{\text {em }}=370-374 \mathrm{~nm}\right)$ e III $\left(\lambda_{\mathrm{em}}=380-384 \mathrm{~nm}\right), \mathrm{I}_{\mathrm{I}} / \mathrm{I}_{\mathrm{III}}$, para vários tipos de náilons estão mostradas na Tabela 1 . Tais valores representam uma média em duplicata. Desses dados, verifica-se que, entre os náilons do tipo $\mathrm{AB}$ (náilons-6 e -11), aquele com menor comprimento de cadeia alifática (náilon-6) apresenta maior polaridade. Da mesma forma, para os náilons do tipo AABB (náilons-6,6, -6,10 e -6,12), a maior polaridade corresponde àquele que possui menor segmento de cadeia proveniente do ácido dicarboxílico original (náilon-6,6).
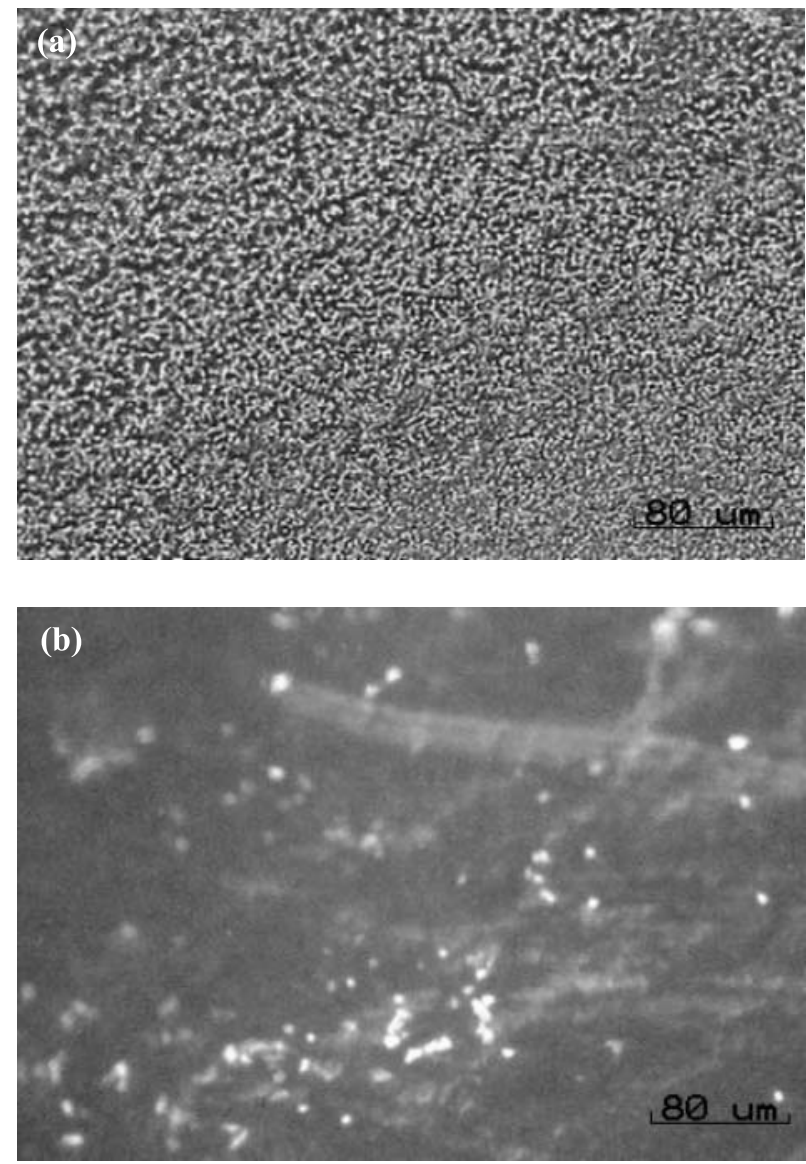

Figura 1. Micrografias ópticas de fluorescência de uma amostra de náilon (a) e de outra amostra contendo pireno adsorvido em superfície (b). 


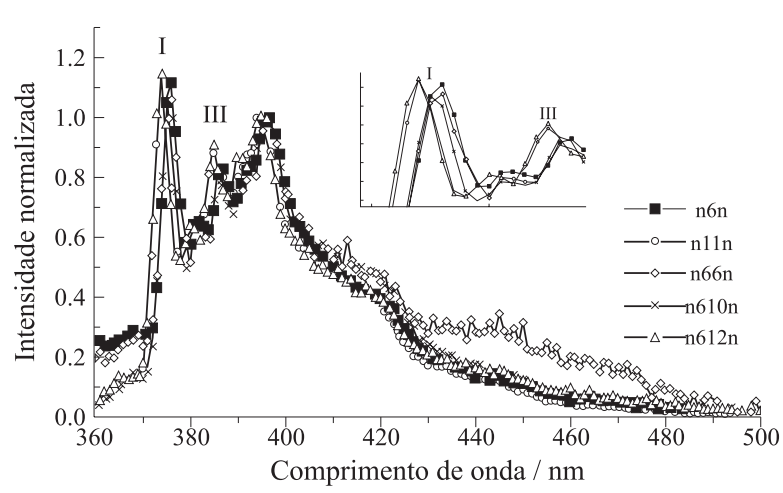

Figura 2. Espectros de fluorescência fotoestacionária do pireno em diferentes tipos de náilons. A intensidade foi normalizada em relação à intensidade da banda III $(385 \mathrm{~nm}) \cdot \lambda_{\mathrm{exc}}=337 \mathrm{~nm}$.

Tabela 1. Relação $\mathrm{I}_{\mathrm{I}} \mathrm{I}_{\mathrm{III}}$ e tempo de vida (com desvio padrão, em ns, para os vários náilons)

\begin{tabular}{ccc}
\hline Náilon & Relação $\mathbf{I}_{\mathrm{I}} \mathbf{I}_{\mathrm{III}}$ & $\tau(\mathbf{n s})$ \\
\hline-6 & 1,41 & $260 \pm 1$ \\
-11 & 1,27 & $276 \pm 3$ \\
$-6,6$ & 1,44 & $250 \pm 3$ \\
$-6,10$ & 1,30 & $296 \pm 3$ \\
$-6,12$ & 1,29 & $310 \pm 3$ \\
\hline
\end{tabular}

O tempo de meia vida do decaimento de fluorescência do pireno também depende da polaridade do meio em torno da sonda. Um exemplo típico desses sinais é mostrado na Figura 3 para o pireno adsorvido em náilon-6. Nessa figura estão mostrados o pulso da lâmpada (pulso rápido), que excita a amostra, e o sinal de decaimento. Após esse pulso, inicia-se a coleta do sinal de emissão da amostra (decaimento mais lento). Após a deconvolução dos dois sinais (eq. 1), o sinal de decaimento é simulado pela função multiexponencial (eq. 2), determinando-se o tempo de meia vida da amostra. Os valores para o pireno adsorvido nos diversos tipos de nái-

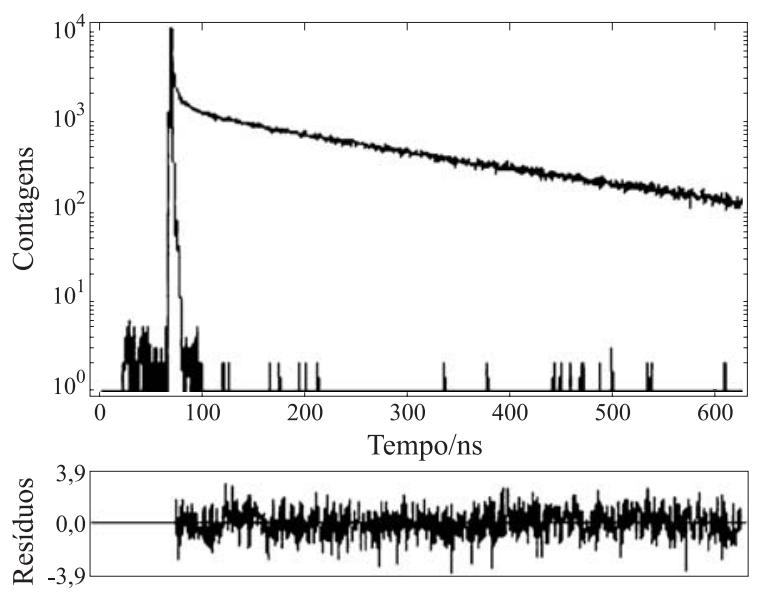

Figura 3. Curva de decaimento de fluorescência do pireno adsorvido na superfície do náilon-6 (superior) e curva de distribuição de resíduos do cálculo de deconvolução em relação ao sinal de decaimento da lâmpada.

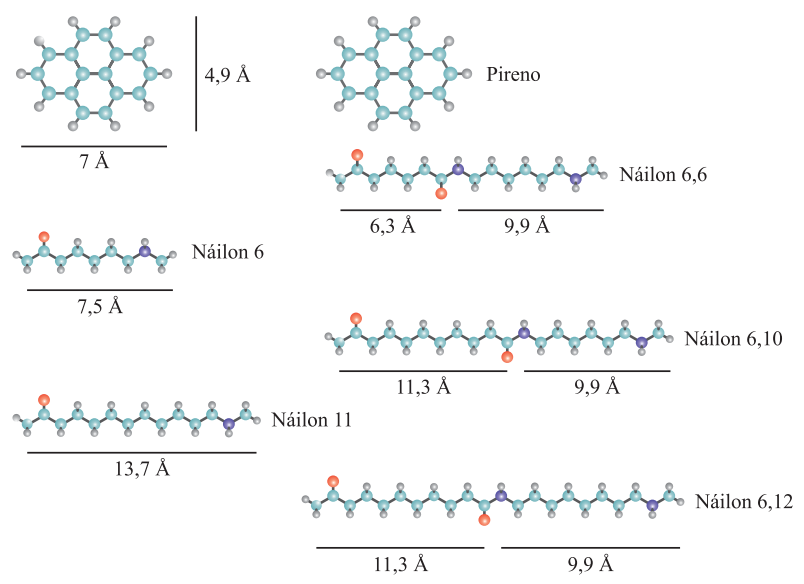

Figura 4. Dimensões relativas da molécula de pireno comparada a dos náilons.

lons estão na Tabela 1. Os decaimentos se mostraram tipicamente como funções monoexponenciais, demonstrando que as moléculas de pireno não interagem entre si, que não há formação de mais que uma camada de pireno adsorvido e que, na média, os sítios de adsorção são similares entre si.

O náilon, sendo obtido por condensação, tem segmentos de cadeia com seqüências e tamanhos bem definidos. Métodos de cálculos de orbitais moleculares semiempíricos ${ }^{[33]}$ foram empregados para se determinar o comprimento máximo dos segmentos das cadeias, assumindo-os como segmentos lineares envolvendo a menor unidade estrutural representativa de cada tipo de náilon. Os resultados dos cálculos estão mostrados em escala relativa na Figura 4, para comparação entre as dimensões relativas da molécula de pireno com uma unidade repetitiva de cada náilon.

Tendo como base as dimensões do eixo molecular mais longo do pireno ( $7 \AA$ ) e a Tabela 1, verifica-se que quando a dimensão do segmento da cadeia linear do náilon é muito maior do que $7 \AA$, sendo: $11,3 \AA$ para o náilon- 6,$10 ; 13,8 \AA$, para o náilon-6,12 e 13,7 Å para o náilon-11 (Figura 4), não mais são observadas mudanças na razão $\mathrm{I}_{\mathrm{I}} / \mathrm{I}_{\mathrm{III}}$ ou nos tempos de decaimentos. Esses resultados mostram que a sensibilidade do pireno à polaridade desses meios está diretamente relacionada com essas dimensões e que, portanto, sua sensibilidade é de curta distância.

As cadeias em um náilon são unidas por ligações de hidrogênio ${ }^{[31]}$. Essas ligações intercadeias geram uma estrutura

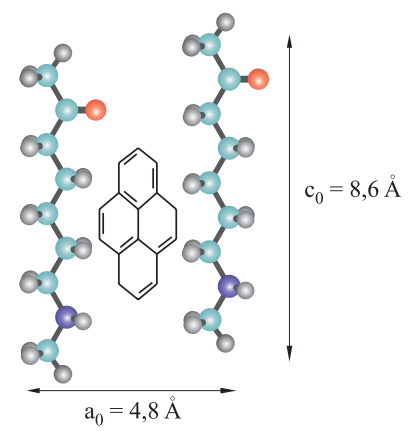

Figura 5. Dimensões da caixa formada por cadeias de náilon- $6^{[33]}$, na qual se alojaria uma molécula de pireno. 
supramolecular na qual duas cadeias de náilon-6 estão separadas por uma distância intercadeia de aproximadamente $4,8 \AA^{[35]}$ (Figura 5), que equivale aproximadamente ao comprimento do eixo curto da molécula de pireno (4,9 $\AA$ ). Podese deste modo propor, com base no esquema da figura 5 , que as dimensões mostradas equivalem a uma "caixa" na qual a molécula de pireno pode ser adsorvida. No caso particular do náilon-6, este pode ser considerado uma matriz Shpol'skii para sonda, isto é uma matriz que, por apresentar cavidades com dimensões próximas às do adsorvato, produzem um espectro de fluorescência com máxima resolução espectral[ ${ }^{[6,22]}$. Em outras palavras, há boa correspondência entre o tamanho e a forma da "gaiola de solvente" e as dimensões moleculares da molécula-hóspede. Neste caso os grupos polares da matriz estão o mais próximos possível das moléculas de pireno. No caso do náilon-11, cujo monômero constituinte tem um comprimento linear muito maior que a da molécula-hóspede (pireno) os grupos polares estão mais afastados e o ambiente no qual a molécula-hóspede se encontra é, portanto, menos polar. Isso se reflete nos valores da relação de intensidades e no tempo de decaimento.

No caso de náilons $\mathrm{AABB}$, a análise é semelhante. As cadeias carboxílicas do náilon-6,6 formam uma caixa de dimensões equivalente às do pireno, podendo este também ser considerado matriz do tipo Shpol'skii. A polaridade desse náilon, inclusive, é similar à do náilon-6. Aumentando-se o tamanho da cadeia carboxílica (do náilon-6,6 para o náilon6,12 , passando pelo $-6,10$ ), mantendo constante o tamanho da componente amida (Figura 4), o comprimento da cadeia alifática se torna maior do que o comprimento do eixo mais longo do pireno, que passa a estar em um ambiente menos polar. Se esse comprimento se torna maior do que um certo valor crítico, o pireno passa a ser pouco sensível a mudanças do meio.

\section{Conclusões}

Os resultados mostram que tanto o espectro de fluorescência quanto o tempo de meia vida do pireno são propriedades fotofísicas que dependem da polaridade dos náilons, da mesma forma que em outros tipos de sistemas. Os das razões de intensidade $\mathrm{I}_{\mathrm{I}} \mathrm{I}_{\mathrm{III}}$ e os tempos de decaimento foram classificados em ordem crescente de polaridade de acordo com a família a que pertencem (AB ou AABB). Tempos de vida menores foram obtidos para os náilons mais polares das duas famílias. Em função da relação de tamanhos da molécula de pireno e dos segmentos das cadeias de náilon, pôde-se concluir que o pireno é um sensor de polaridade de curto alcance, ou seja: acima de uma certa distância as suas propriedades fotofísicas são invariantes com a polaridade do meio.

\section{Agradecimentos}

Os autores agradecem à FAPESP, ao CNPq e ao MCT/ PADCT/IMMP pelos auxílios à pesquisas e ao CNPq pela bolsa de pesquisador.

\section{Referências Bibliográficas}

1. Tonelli, A. E. - "NMR Spectroscopy and Polymer Microstructure: The Conformational Connection", VCH Publ., New York (1989).

2. Koenig, J. L. - "Spectroscopy of Polymers", $2^{\text {nd }}$ ed., Elsevier, New York (1999).

3. Kamioka, K. \& Webber, S. E. - Chem. Phys.Lett., 133, p.353 (1986).

4. Yu, Z.; McGervey, J. D.; Jamieson, M. A.\& Simba, R. Macromolecules, 28, p.6268 (1995).

5. do Nascimento, G. M.; Pereira da Silva, J. E. \& Córdoba de Torresi, S. I. - Macromolecules, 35, p.121 (2002).

6. Lakowickz, J. R. - "Principles of Fluorescence Spectroscopy", $2^{\text {nd }}$ ed., Kluwer Academic \& Plenum Publications, New York (1999).

7. M. A. Winnik - (ed.) "Photophysical and Photochemical Tools in Polymer Science: conformation, dynamics and morphology", NATO ASI Series, V. 183, Riedel, Dordrech (The Netherlands) (1986).

8. Santos, Jr., D. S.; Mendonça, C. R.; Balogh, D. T.; Dhanabalan, A.; Giacometti, J. A.; Zílio, S. C. \& Oliveira, Jr., O. N. Synthetic Metals, 121, p. 1479 (2001).

9. Mendonça, C. R.; Santos, Jr., D. S.; Balogh, D. T.; Dhanabalan, A.; Giacometti, J. A.; Zílio, S. C. \& Oliveira, Jr., O. N. - Polymer, 42, p.6539 (2001).

10. Itagaki, H.; Horie, K. \& Mita, I. - Progr. Polym. Sci., 15, p. 361 (1990).

11. Zimerman, O. E. \& Weiss, R. G. - J. Chem. Phys., 102, p.5364 (1998).

12. Prado, E. A.; Yamaki, S. B.; Atvars, T. D. Z.; Zimerman, O. E. \& Weiss, R. G. - J. Phys. Chem. B, 104, p.5905 (2000).

13. de Andrade, M. L. \& Atvars, T. D. Z. - J. Phys. Chem. B: 2004 (in press).

14. Deus, J. F.; de Andrade, M. L.; Atvars, T. D. Z. \& Akcelrud, L. - Chem. Phys., 297, p.177 (2004).

15. Coltro, L.; Dibbern-Brunelli, D.; Elias, C. A. B.; Talhavini, M.; Oliveira, M. G. \& Atvars, T. D. Z. - J. Braz. Chem. Soc., 6, p.127 (1995).

16. Talhavini, M.; Atvars, T. D. Z.; Schurr, O. \& Weiss, R. G. - Polymer, 39, p.3221 (1998).

17. Vigil, M. R.; Bravo, J.; Atvars, T. D. Z. \& Baselga, J. Macromolecules, 30, p.4871 (1997).

18. Vigil, M. R.; Bravo, J.; Atvars, T. D. Z. \& Baselga, J. Curr. Org. Chem., 7, p.197 (2003).

19. Thomas, J. K. - J. Phys. Chem., 91, p.267 (1987). 
20. Nathanson, A. - Chem. Rev., 102, p.4159 (2002).

21. Turro, N. J. - “Modern Molecular Photochemistry”, Univ. Science Books, Sausalito (1991).

22. Birks, J. B. - "Photophysics of Aromatic Molecules", John Wiley \& Sons, London (1970).

23. Venkataraman, K. - "The Chemistry of Synthetic Dyes", vol. 1, Academic Press, New York (1952).

24. Rys, P. \& Zollinger, H. - "Fundamentals of the chemistry and application of dyes", Wiley-Interscience, London (1972).

25. Canning, A. J.; Jarman, C. G. \& Mykoluk, S. - "Pad-batch dyeing of plant fibres", Tropical Products Institute, London (1977).

26. Timofei, S.; Schmidt, W.; Kurunczi, L. \& Simon, Z. Dyes and Pigments, 47, p.5 (2000).

27. Sokolovski, P.; Garcia, C. M. \& Atvars, T. D. Z.; - "Estudos Espectrofotométricos da Adsorção de Corantes e de Branqueantes Ópticos em Fibras de Viscose", in: Anais do 7-Congresso Brasileiro de Polímeros (CD), Belo Horizonte - MG, out. (2003).
28. Kalyanasundaram, K. \& Thomas, J. K. - J. Am. Chem. Soc., 99, p.2039 (1977).

29. Dong, D. C. \& Winnik. M. A. - Can. J. Chem., 62, p. 2560 (1984).

30. Jay, J.; Johnston, L. J. \& Scaiano, J. C. - Chem. Phys. Lett., 148, p.517 (1988), (b) Roelants, E.; de Schryver, F. C. - Langmuir, 3, p.209 (1987).

31. Kaufman, V. R. \& Avnir, D. - Langmuir, 2, p.717 (1986).

32. Kohan, I. - "Nylon Plastics Handbook", Hanser/Gorden Publ., Inc., Cincinnati (1995).

33. Schleyer, P. V. R. (ed.) - "Encyclopedia of Computational Chemistry", John Wiley \& Sons, Chichester (1998).

34. Cruz, M. C. P.; Cassiola, F. M. \& Atvars, T. D. Z. - J. Appl. Polym. Sci., 84, p.1637 (2002).

35. Léon, S.; Alemán, C. \& Muñoz-Guerra, S. Macromolecules, 33, p.5754 (2000).

Enviado: $30 / 04 / 04$

Reenviado: $17 / 08 / 04$

Aprovado: 22/09/04 